\title{
On the Use of SOA-Based Tunable Dispersion Compensator in Ultrafast Incoherent Fiber-Optic CDMA Systems Under Temperature Variation
}

\author{
Wing C. Kwong, Senior Member, IEEE, Saleh Seyedzadeh*, Member, IEEE, \\ Ivan Glesk*, Senior Member, IEEE, and Guu-Chang Yang**, Fellow, IEEE \\ Department of Engineering, Hofstra University, Hempstead, NY 11549, USA \\ * Faculty of Engineering, University of Strathclyde, Glasgow G1 1XW, UK \\ ** Department of Electrical Engineering and Graduate Institute of Communication Engineering, \\ National Chung Hsing University, Taichung 402, Taiwan
}

\begin{abstract}
The use of semiconductor-optical-amplifier-based tunable dispersion compensator (SOA-TDC) in ultrafast incoherent fiber-optic code-division multiple-access (FO-CDMA) systems with picosecond multiwavelength codes has recently been demonstrated. In this paper, results on the SOA-TOC's capability of compensating for fiber chromatic dispersion and distorted autocorrelation function caused by fiber temperature variation (FTV) in such a FO-CDMA system with a long fiber link are reported. The deleterious effects of FTV to the system performance are quantified in terms of "chip granularity" and studied by applying a recent multiple-QoS performance-analytical model.
\end{abstract}

Keywords: code division multiple access, dispersion compensation, optical fiber communications, quality of service, temperature variation.

\section{INTRODUCTION}

The deleterious effects of fiber temperature variation (FTV) to ultrafast incoherent fiber-optic code-division multiple-access (FO-CDMA) systems using picosecond multiwavelength codes have recently been studied [1][5]. Causing dispersion and time skew to these multiwavelength codes, FTV can distort the auto- and crosscorrelation functions seen at receivers and thus create detection errors in transmitted data bits [6], [7]. In addition, chromatic dispersion (CD) accumulated in a long fiber link requires the application of dispersion compensating fiber (DCF). To fully compensate for CD, precise setup of the DCF is needed but rather difficult to achieve in a network. As a result, small amount of CD may still exit from the DCF. Besides completely eliminating the residual $\mathrm{CD}$, Ahmed, et al. [3]-[5] proposed a semiconductor-optical-amplifier (SOA)-based tunable dispersion compensator (TDC) to compensate for the FTV distortion to the autocorrelation function, however, not to the cross-correlation function. To account for the effect of the FTV-distorted cross-correlation function (at the SOATDC output) to the performance of this kind of FO-CDMA systems, new analytical models were studied [6], [7].

In this paper, the use of the SOA-TDC in an ultrafast incoherent FO-CDMA system using picosecond multiwavelength codes in a long fiber link is reviewed in Section 2. The deleterious effects of FTV to this system are studied and quantified in terms of "chip granularity" [3]. In Section 3, a recent multiple-quality-of-service (QoS) analytical model [7] is applied to study the performance of this FO-CDMA system with different amounts of FTV (in terms of chip granularity) and QoS (in terms of code weight).

\section{Overviews of SOA-TDC and Fiber Temperature Variation}

In an ultrafast incoherent FO-CDMA system [8]-[10], if an arrival codeword of weight $w$ (i.e., number of multiwavelength pulses) matches with the address (codeword) signature of a receiver, these $w$ pulses will ride atop each other within one chip. This creates an autocorrelation peak of height $w$, and a data bit of 1 will be recovered correctly if there is no CD or FTV distortion. Besides residual CD exiting from the DCF, FTV distortion (in forms of dispersion and time skew) will be imposed to these mutliwavelength pulses if there exists temperature difference in the long fiber link between the transmitter and receiver. To achieve precise CD and FTV-distortion compensation in the SOA-TDC [3]-[5], the SOA's input-power sensitivity is tuned to the power level of the autocorrelation peak so that the gain-compression mechanism of the SOA is created accordingly and precisely. Fig. 1 shows the experimental setup of the SOA-TDC for such compensation. A picosecond supercontinuum laser is used to generate optical supercontinuum, which is sliced by a fiber-Bragg-gratings-based O-CDMA encoder [9], [10]. The encoder generates a 4-wavelength codeword of weight $w=4$, based on wavelengths 1,550.12, 1,550.92, 1,551.72, and 1,552.52 nm of each $12 \mathrm{ps}$ (FWHM) in pulse-width. The system's repetition rate is $2.5 \mathrm{GHz}$. These correspond to chip-width of $12 \mathrm{ps}$, code length of 33 chips, and data rate of $2.5 \mathrm{Gbit} / \mathrm{s}$ (i.e., period of $400 \mathrm{ps}$ ). The codewords then travel in a $20-\mathrm{km}$ CD-compensated fiber link with $45{ }^{\circ} \mathrm{C}$ of temperature difference between the 
encoder and decoder. The distorted autocorrelation function at the decoder output is then properly compensated by the SOA-TDC. The compensation results are observed in a sampling oscilloscope. Fig. 2 shows the screen shots of the autocorrelation function before and after compensation by the SOA-TDC, respectively. Because of the sign of the FTV thermal coefficient [3]-[5], Fig. 2a shows the distorted autocorrelation peak being compressed to 8 ps (FWHM). While the SOA-TDC can fully compensate the autocorrelation peak back to 12 ps (FWHM), Fig. 2b illustrates the compensation can be adjusted to any value by showing a compensated peak of 14 ps (FWHM).

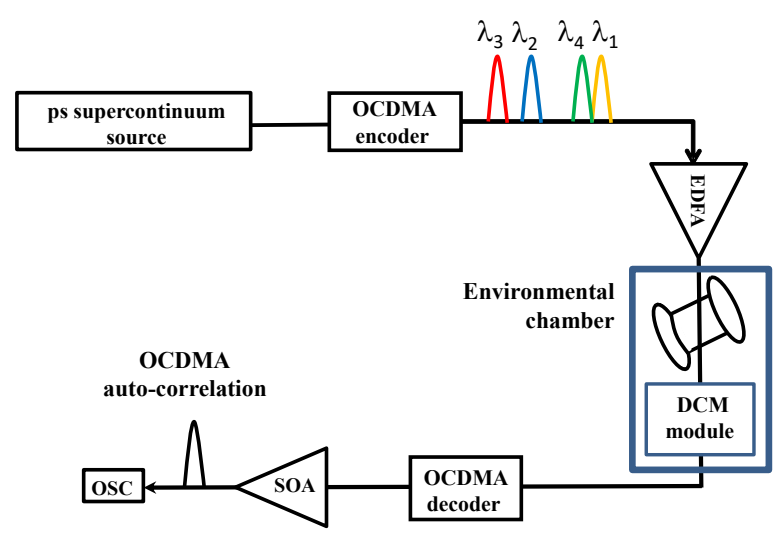

Figure 1. Experimental setup of a FO-CDMA link with the SOA-TDC. (OSC-oscilloscope, DCM-dispersion compensating module, and EDFA-Erbium-doped fiber amplifier.)
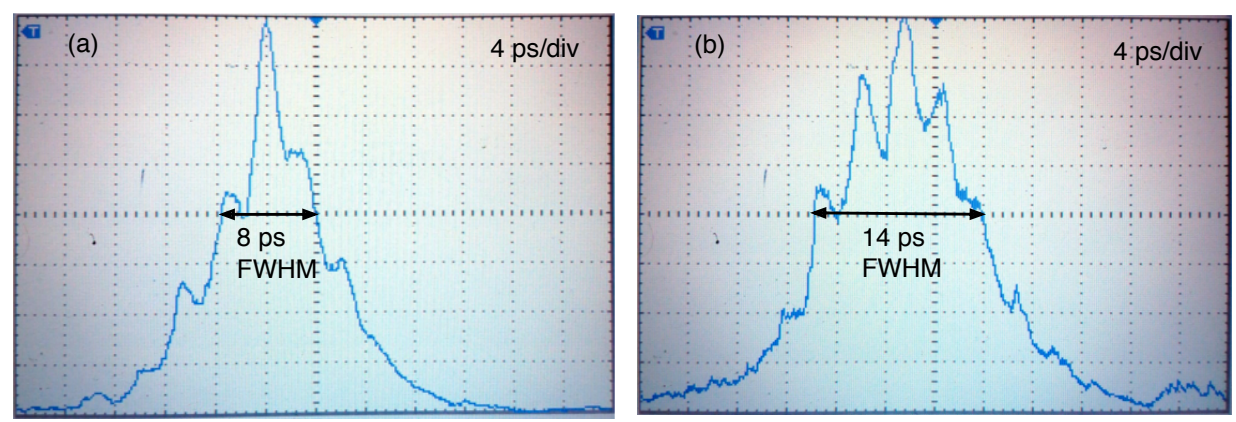

Figure 2. (a) A distorted autocorrelation peak of 8 ps (FWHM) after a 4-wavelength codeword of $w=4$ with original chip-width of 12 ps (FWHM) being transmitted in a 20-km CD-compensated fiber link with $45^{\circ} \mathrm{C} \mathrm{FTV}$.

(b) A compensated autocorrelation peak of 14 ps (FWHM) after the SOA-TDC.

\subsection{Quantifying Metric for the FTV Effects to Cross-Correlation Function}

While the cross-correlation functions (from interfering codewords) are also distorted by the FTV time skew and dispersion [6], [7], they generally have much lower power and thus fail to trigger the gain-compression mechanism of the SOA-TDC. The deleterious effect of these distorted cross-correlation functions to the ultrafast incoherent FO-CDMA system performance need to be quantified and modelled accordingly.

As studied [6], [7], the envelope of the $k$ th wavelength of the multiwavelength pulses from the picosecond supercontinuum laser under FTV can be modelled as

$$
S_{k}(t)=\frac{\sigma}{\sigma-\Delta \sigma} \operatorname{sech}^{2}\left(\frac{t-k \Delta t}{\sigma-\Delta \sigma}\right)
$$

with time skew $\Delta t=D_{\text {temp }} \times \Delta T \times \Delta \Lambda \times d(\mathrm{ps})$ and dispersion $\Delta \sigma=D_{\text {temp }} \times \Delta T \times \Delta \lambda \times d(\mathrm{ps})$ for $k \in[0, w-$ 1], where $D_{\text {temp }}\left(\mathrm{ps} / \mathrm{nm} / \mathrm{km} /{ }^{\circ} \mathrm{C}\right)$ is the FTV thermal coefficient and $\Delta T\left({ }^{\circ} \mathrm{C}\right)$ is the temperature difference in a fiber link of $d(\mathrm{~km})$. For these multiwavelength pulses, each wavelength has spectral linewidth $\Delta \lambda(\mathrm{nm})$, is equally spaced by $\Delta \Lambda(\mathrm{nm})$, and carries FWHM width (i.e., chip-width) $t_{c} \approx 1.76 \sigma$, where $\sigma$ is defined as the $\operatorname{sech}^{2}$ pulse duration.

To illustrate the FTV effect to the cross-correlation function, carrier-hopping prime codes (CHPCs) of 31 chips and $w=4$ with four wavelengths are used in this paper [9], [10]. Also assume that $t_{c}=10$ ps (i.e., $\sigma=5.68$ ), $\Delta \lambda=1 \mathrm{~nm}, \Delta \Lambda=1 \mathrm{~nm}, D_{\text {temp }}=0.0014 \mathrm{ps} / \mathrm{nm} / \mathrm{km} /{ }^{\circ} \mathrm{C}, \Delta T=45^{\circ} \mathrm{C}$, and $d=20 \mathrm{~km}$. These parameters give $\Delta t=$ $1.26 \mathrm{ps}, \Delta \sigma=1.26 \mathrm{ps}$, (dispersed) pulse width of $t_{c}=1.76(\sigma-\Delta \sigma)=7.78 \mathrm{ps}$ (FWHM), and time skew of the $k$ th wavelength pulse $k \Delta t=1.26 k$ ps for $k \in[0,3]$. As a result, these multiwavelength pulses are regularly timeskewed as a function of $\Delta t$, and the time skew can be modelled in term of chip granularity $g=\llbracket t_{c} / \Delta t \rrbracket$ by subdividing each chip-width $t_{c}$ into sub-chip-width $\Delta t$, where $\llbracket \cdot \rrbracket$ is a rounding function [6]. With $\Delta \lambda=\Delta t=1.26$ 
ps and $t_{c}=10 \mathrm{ps}$, this example can be treated as a time-skew model of $g=8$. The time skew will cause these multiwavelength pulses to partially fall into adjacent chips and thus create stronger multiaccess interference (MAI) in the cross-correlation function. As the cross-correlation function gets worsened (i.e., larger), the auto-to-crosscorrelation ratio gets reduced and so is the system performance, even though the SOA-TDC is used to fully recover the autocorrelation peak. As shown in Section 3, the use of $g$ to quantify the FTV effect to the cross-correlation function is an important parameter in determining the performance of such a FO-CDMA system.

\section{Adjustable-QoS System Performance Analysis}

To account for the actual effect of FTV under the SOA-TDC, the adjustable-QoS, hard-limiting error probability (i.e., performance-analytical model) of the CHPCs in an incoherent FO-CDMA system with on-off-keying modulation has been formulated as [7]

$$
\mathrm{Pe}=\frac{1}{2} \sum_{i=0}^{w}(-1)^{w-i}\left(\begin{array}{c}
w \\
i
\end{array}\right)\left[q_{0}+\frac{i}{w} q_{1}+\frac{i(i-1)}{w(w-1)} q_{2}\right]^{K-1}
$$

with the probabilities of getting zero, one, and two hits in the FTV-distorted cross-correlation function given by $q_{0}=1-q_{1}-q_{2}$,

$$
\begin{aligned}
& q_{1}=\frac{[2 w(N-w+1)-N-1] g}{2 N(N-1)(2 g-1)} \\
& q_{2}=\frac{(w-1)^{2}(2 g-1)}{8 N(N-1)(g-1)}
\end{aligned}
$$

respectively, where $K-1$ represents the number of interfering users, $N$ is the number of chips, $w<N$, and $g \geq w$.

Fig. 3 compares the hard-limiting error probability $P$ e, from (2)-(4), of the CHPCs in the adjustable-QoS performance-analytical model with $N=13$ chips, weight $w=\{7,8,9\}$, and chip granularity $g=\{2,3,4,5,8,20\}$. The dashed curves show the $P_{\mathrm{e}}$ of the baseline case of no FTV distortion (i.e., $\Delta t=0$ ). Because $g=\llbracket t_{c} / \Delta t \rrbracket$, this ideal case is equivalent to $g=\infty$ and gives the performance lower bound. The $P_{\text {e }}$ generally gets worsened as the number of simultaneous users $K$ increases because more interferers will create stronger MAI and thus worsen the cross-correlation function. On the other hand, the $P_{\mathrm{e}}$ improves with $w$ because heavier code weight increases the autocorrelation-peak's height for better MAI discrimination. By varying $w$, the QoS of the system can be adjusted. With the same $w$, the $P_{\mathrm{e}}$ improves with $g$ and will approach to that of the ideal $g=\infty$ case as $g$ gets larger. Because the FTV time skew is a function of $\Delta t$, chip granularity $g=\llbracket t_{c} / \Delta t \rrbracket$ gets smaller when the FTV effect is worsened (i.e., $\Delta t$ gets larger). A larger $\Delta t$ (i.e., smaller $g$ ) means that more portions of MAI will fall into adjacent chips in the cross-correlation function, resulting in larger hit probabilities and, in turn, worsening system performance. In other words, as the FTV effect gets lessened, a larger $g$ value indicates smaller amount of MAI and thus better system performance.

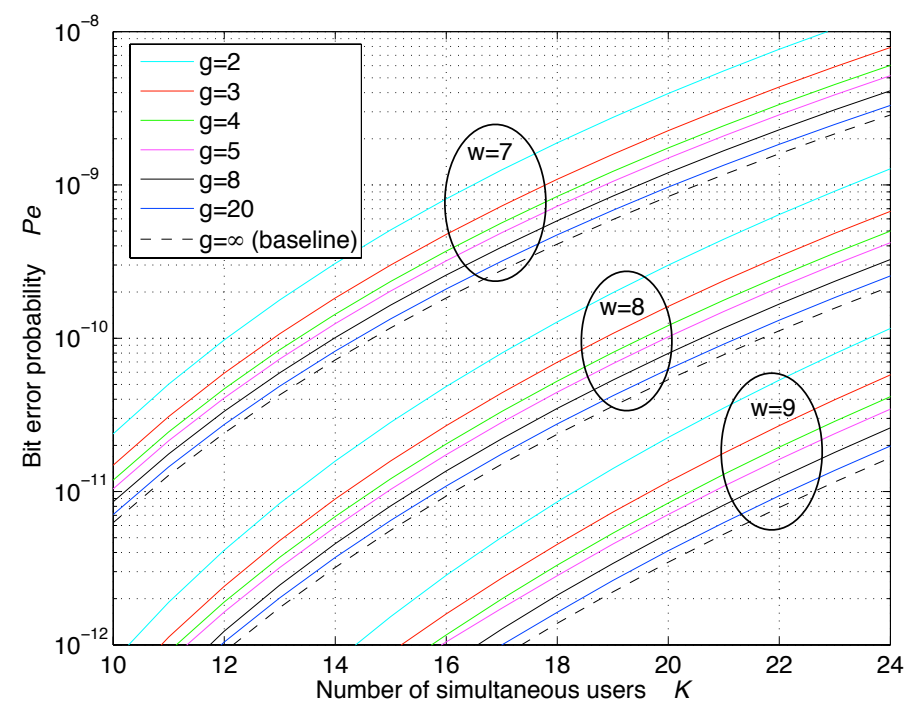

Figure 3. Harding-limiting bit error probability $P_{\mathrm{e}}$ of the CHPCs in the adjustable-QoS performance model versus the number of simultaneous users $K$ with $N=13$ chips, $w=\{7,8,9\}$, and $g=\{2,3,4,5,8,20\}$.

\section{CONCLUSIONS}

The use of the SOA-TDC in an ultrafast incoherent FO-CDMA system with picosecond multiwavelength codes in a long fiber link under the influence of FTV was reviewed. The FTV effects to such a FO-CDMA system were overviewed and modelled in term of chip granularity. An adjustable-QoS performance-analytical model was applied to quantify the system performance with different amounts of FTV (in terms of chip granularity) and QoS 
(in terms of code weight). With the use of the SOA-TDC to recover the original autocorrelation function, the study showed that the FTV effect to the cross-correlation function could not be ignored and, in fact, should be related to chip granularity. A lessened FTV effect was reflected by larger chip granularity, which, in turn, gave better system performance.

\section{ACKNOWLEDGEMENTS}

This work was supported in part by the Faulty Development and Research Grants of Hofstra University, in part by the Ministry of Science and Technology of Republic of China under Grant MOST 105-2221-E-005-007-MY3, and in part by the European Union's Horizon 2020 research and innovation program under the Marie SklodowskaCurie grant agreement No 734331.

\section{REFERENCES}

[1] T.B. Osadola, S.K. Idris, I. Glesk, and W.C Kwong, "Effect of variations in environmental temperature on 2D-WH/TS OCDMA code performance,” J. Opt. Commun. Netw., vol. 5, no. 1, pp. 68-73, Jan. 2013.

[2] C.-Y. Tsai, G.-C. Yang, J.-S. Lin, C.-Y. Chang, I. Glesk, and W.C. Kwong, "Pulse-power-detection analysis of incoherent O-CDMA systems under the influence of fiber temperature fluctuations," J. Lightw. Technol., vol. 35, no. 12, pp. 2366-2379, Jun. 2017.

[3] M.S. Ahmed, M.S.K. Abuhelala, and I. Glesk, "Managing dispersion-affected OCDMA auto-correlation based on PS multiwavelength code carriers using SOA," J. Opt. Commun. Netw., vol. 9, no. 8, pp. 693-698, Aug. 2017.

[4] M.S. Ahmed and I. Glesk, "Mitigation of temperature-induced dispersion in optical fiber on OCDMA autocorrelation," IEEE Photon. Technol. Lett., vol. 29, no. 22, pp. 1979-1982, Nov. 15, 2017.

[5] M.S. Ahmed and I. Glesk, "Management of OCDMA auto-correlation width by chirp manipulation using SOA," IEEE Photon. Technol. Lett., vol. 30, no. 9, pp. 785-789, May 2018.

[6] Y.-T. Lin, G.-C. Yang, C.-Y Chang, and W.C. Kwong, "Design and analysis of asynchronous incoherent optical-CDMA systems using a new code-shifting technique," IEEE Trans. Commun., vol. 63, no. 7, pp. 2619-2631, Jul. 2015.

[7] C-W. Chang, G.-C. Yang, I. Glesk, and W.C. Kwong, "On the performance of the effects of temperature variation in ultrafast incoherent fiber-optic CDMA systems with SOA-based tunable dispersion compensator," IEEE Photon. J., under review.

[8] W.C. Kwong, P.A. Perrier, and P.R. Prucnal, "Performance comparison of asynchronous and synchronous code-division multiple-access techniques for fiber-optic local area networks," IEEE Trans. Commun., vol. 39, no. 11, pp. 1625-1634, Nov. 1991.

[9] G.-C. Yang and W.C. Kwong, Prime Codes with Applications to CDMA Optical and Wireless Networks, Norwood, MA: Artech House, 2002.

[10] W.C. Kwong and G.-C. Yang, Optical Coding Theory with Prime, New York: CRC Press, 2013. 